\title{
A parallel machine extension to aversion dynamics scheduling
}

\author{
Gary W. Black ${ }^{\mathbf{a}^{*}}$ and Kenneth N. McKay
}

${ }^{a}$ College of Business, University of Southern Indiana, 8600 University Blvd, Evansville, IN 4771, USA

${ }^{b}$ Department of Management Sciences, University of Waterloo, 200 University Avenue W, Waterloo, ONT, N2L 3G1, Canada

AR T I CLEIN F O ABS TRAC T

\begin{tabular}{l}
\hline Article history: \\
Received 31 January 2012 \\
Accepted March, 72012 \\
Available online \\
8 March 2012 \\
\hline Keywords: \\
Aversion dynamics \\
Scheduling \\
Risk mitigation \\
Safety time \\
Job re-sequencing
\end{tabular}

The aversion dynamics research agenda has incorporated within dispatching heuristics a number of real-world observations involving risk mitigation practices used by real schedulers. One such observation is that schedulers occasionally offload risky jobs from a primary machine to otherwise less desirable machine (older, slower) during periods of peak load to avoid the effects the risky job can have on subsequent jobs. This paper examines this situation within the proportional parallel machine environment. Safety time is used to adjust dispatching priorities of risky jobs to reflect the aversion. The effect of various safety time values on performance is studied. Robust safety time values and/or intervals are identified across a variety of experimental factors related to risk level, percent risky jobs in the job stream, and due date distribution.

(C) 2012 Growing Science Ltd. All rights reserved

\section{Introduction}

The gap between scheduling research and practice has inspired an area that has become known as aversion dynamics (McKay et al. 2000; McKay and Black, 2006). Aversion dynamics is based on empirical field studies which reveal how production schedulers incorporate aversion to risk within their daily decisions (McKay, et al. 1995; McKay, 1992). For instance, suppose a scheduler delays assigning a sensitive job to a risky machine until such time the risk subsides. An example of this situation is the period of instability shortly after a major machine upgrade or breakdown. Another example is when a scheduler delays assigning a risky job, perhaps a prototype part, to a machine due to the potential effect it can have on subsequent jobs; the prototype part could destabilize the process, machine settings, or possibly damage the machine. When these strategies are incorporated into the aversion dynamic heuristics, the processing time used for prioritization has been inflated beyond the nominal time to reflect the risk (a form of surrogate for the risk assessment), thus affecting sequencing decisions.

To model such risk mitigation strategies, several aversion heuristics have been developed. Averse-1, the initial heuristic, applies to sensitive jobs on risky machines in the single-machine, static arrival

* Corresponding author. Tel: +1(812) 461-5385

E-mail: gblack@usi.edu (G. Black)

(C) 2012 Growing Science Ltd. All rights reserved. doi: $10.5267 /$ j.ijiec.2012.03.002 
environment (McKay et al. 2000). It only considers the timeframe after the disruptive event and does a form of selective sub-optimization during the risk interval, returning to a normal state as the risk reduces. Averse- 2 extended aversion dynamics to the single-machine, dynamic arrival scenario and considered the timeframe both before (proactive) and after (reactive) the disruptive event (Black et al. 2004; Black, 2001). This allowed work to be pulled ahead of the risk period to mitigate potential damage, as well as alter the sequencing after the potentially risk event. Averse-3 further extended the problem structure to reduce the potential for in-process jobs at the time of the risk event (Black et al. 2005). Other aversion dynamics heuristics have been developed as well, such as risky jobs on sensitive machines (Black et al. 2006), as opposed to sensitive jobs on risky machines. Moreover, anticipatory batch insertion has extended aversion dynamics from the sequencing environment to the lot sizing environment to study the effect of running a small test batch in advance of the main batch to absorb instability from the risk and to stabilize the process prior to running the main batch (Black et al. 2008).

Empirical studies have also shown risk aversion to be practiced within the parallel-machine environment (Aytug et al. 2005). For example, a risky job may be offloaded from a primary machine to an otherwise less desirable (e.g., slower) secondary machine to reduce the disruptive effects it can have on subsequent jobs. For example, prototype jobs often require debugging at the machine before they run efficiently. If such jobs are processed on the primary machine, they can delay subsequent jobs considerably. Offloading them to a secondary, albeit slower, machine (e.g., "prototype machine") is often employed in practice. Consequently, this paper will extend aversion dynamics from the singlemachine to the parallel-machine environment to study this situation. Performance will be examined across a variety of cases involving risk occurrence and level, "safety time" values used in job priorities, job stream composition and due date levels.

\section{Literature Search}

Various review papers have examined the gap between scheduling research and practice (Aytug et al. 2005; McKay et al. 2002; McKay and Wiers, 1999). A variety of machine configurations, performance measures, dispatching heuristics and arrival patterns have been developed and studied in detail. However, the application of theory in practice is still quite limited. A key reason is the lack of fidelity of the scheduling problem being modeled. Real-world production environments are not static and predictable, but rather dynamic and uncertain. Stochastic scheduling and robust/rescheduling techniques attempt to model such variability to an extent. For example, Dubois et al. (2003) and Kleijnen and Gaury (2003) examined methods for modeling uncertainty. Shafaei and Brunn (1999, 2000) examined schedule robustness measures under uncertainty. Cowling and Johansson (2002) and Ovackik and Uzsoy $(1994,1995)$ presented techniques for constructing robust schedules. Kanet and Sridharan (2000) and O'Donovan et al. (1999) introduced idle time/schedule slack insertion to absorb variability. However, all such research except O'Donovan et al. (1999) assumed variability is job independent and, thus, applied more to routine manufacturing noise (i.e., random variability) than to any job-dependent phenomena. As review articles have suggested, this assumption is pervasive throughout the literature with few exceptions (Aytug et al. 2005, Cao et al. 2001).

The empirically inspired area of aversion dynamics has introduced job and time dependency into the scheduling problem. Several long-term, longitudinal field studies have shown that $10-15 \%$ of scheduling decisions relate to job-dependent issues beyond routine manufacturing noise (McKay, et al. 1995; McKay, 1992). Some risks are predictable in advance (e.g., machine upgrades, engineering changes) and can be proactively addressed; other risks are unpredictable and must be reacted to (e.g., machine breakdowns). Ideally, the production environment after the risk event ends should be the same as beforehand (i.e., time independent). However, McKay, et al. (1995) showed this is often not true. For example, after a machine is repaired, it often requires further time (e.g., calibration, adjustment) until its performance is restored to $100 \%$ of its pre-event level. Further, some jobs are more sensitive to these lingering impacts than other jobs. To study this problem of sensitive jobs on 
risky machines, the Averse- 1 heuristic was developed for the single-machine, static-arrival environment (McKay et al. 2000). Priorities of sensitive jobs were altered after the risk event using a time-based decay principle which resulted in sensitive jobs being delayed in the sequence until such time that the potential impacts associated with the risk declined and performance was restored to its pre-event level. Results were favorable, indicating significant performance gains when the impact occurred as predicted and insignificant losses when it did not.

Although Averse-1 introduced job dependency and time dependency, it had several limitations. It only considered the static job arrivals and the time after the risk event (i.e., lingering impacts). Consequently, the Averse-2 heuristic was developed to consider dynamic job arrivals and the time before the risk event (Black et al. 2004; Black, 2001), thus making it possible to apply aversion dynamics in a proactive manner before a perceived risk event (e.g., re-sequencing sensitive jobs in anticipation of a pending machine upgrade). Results again indicated significant performance gains when the risk and impact occurred as predicted and insignificant losses when they did not.

Although Averse-2 attempted to minimize post-event impacts by proactively and reactively resequencing sensitive jobs, it made no attempt to avoid an in-process job at the time when the risk event occurs. In-process jobs result in a fragmented processing time profile. Further, they often need to be scrapped and/or the machine needs to be set up a second time for the same job after the risk event. McKay (1992) showed that schedulers seek to avoid such fragmentation in one of two ways. They may intentionally hold the machine idle when a disruption is considered imminent, or they may schedule a lower priority job to bridge the gap between the current time and predicted event time. Consequently, the Averse-3 heuristic was developed (Black et al. 2005). Linear and exponential priority reduction profiles and a logical constraint were examined in an attempt to minimize in-process jobs. Results showed that fragmentation can be significantly reduced with only minimal impact on weighted tardiness performance.

Aversion dynamics research next considered the complementary problem, namely risky jobs on sensitive machines. Here, the jobs themselves are the risk entities (instead of the machine). Real schedulers have been observed to be averse to scheduling risky jobs on highly loaded (sensitive) machines (Aytug et al. 2005), instead preferring to:

Case 1: Schedule them during quieter (more lightly loaded) periods, or

Case 2: Offload them to an otherwise less desirable machine (e.g., older, slower machine).

Case 1 pertains to the single-machine environment and was investigated by Black et al. (2006). "Safety time" was used to adjust job priorities of risky jobs in order to smooth the effects of processing time variability. Robust safety time values were identified for various objective functions (maximum tardiness, weighted flow time and weighted tardiness) and various experimental conditions related to risk level and due date distribution.

Case 2 pertains to the parallel machine environment and is the subject of this paper. The methodology used to implement this research is discussed in the next section.

\section{Methodology}

This paper considers a 2-machine proportional parallel machine configuration with a "fast" machine and "slow" machine. The fast machine is the primary machine used to run product. The slow machine is a secondary machine used to supplement the fast machine during periods of high load and also to accommodate risky jobs whenever the schedule is tight. The objective is to examine how various attributes related to job risk, job stream composition and due date affect weighted tardiness performance. Specific variables and settings appear in Section 4. 
Parallel machine models require not only sequencing decisions, but routing decisions as well. Fortunately, this particular configuration can be simplified by taking advantage of the fact that there is really just one input queue whose priority sequence needs to be determined to solve the problem (Morton and Pentico, 1993). There exists an optimal solution of the following form: sequence the $n$ jobs in an optimal order in a list (method for obtaining the optimum order has not yet been specified) and, in turn, assign each job to the machine that can finish it first.

The methodology used to implement this logic within the static arrival, non-preemptive case is as follows. At each sequencing decision point (i.e., when a machine is empty), assign the highest priority job to the machine that will finish it first. Obviously, if the fast machine is empty, assign the job to it since, by definition, it will always finish it first. However, if the slow machine is empty, it may or may not be able to finish that job first due to the speed differential between the (currently busy) fast machine and the (currently empty) slow machine. In such cases, it is necessary to make a series of temporary job assignments to the fast machine until we find the first job that will finish on the slow machine first. Job priorities are recomputed at each decision point and after each temporary assignment. Once the first job is found that will finish first on the slow machine, it is permanently assigned to the slow machine, all temporary assignments are deleted, and the process is repeated to find the next job to include in the sorted list.

Job priorities are computed using the R\&M dispatching rule (Morton et al. 1995; Morton and Pentico, 1993). Also called the "Apparent Tardiness Cost" rule, R\&M is a composite rule combining the weighted shortest processing time (WSPT) rule with an additional term used to modify job priority as a function of processing time slack. Since R\&M results in dynamic job priorities, it has been shown to be effective in dynamic situations involving job risk that changes over time (Morton et al. 1995; Morton and Pentico, 1993). R\&M has the following form:

$$
\pi_{j}=\left(w_{j} / p_{j}^{*}\right) e^{-S_{j}^{+} / k p_{\text {ave }}} \text {. }
$$

The first term, $\pi_{j}=\left(w_{j} / p_{j}{ }^{*}\right)$, is simply the WSPT rule in which $p_{j}{ }^{*}$ represents the planning (estimated) processing time of job $j$ and $w_{j}$ reflects its weight. R\&M appends a second term, $e^{-S_{j}{ }^{+} / k p_{\text {ave }}}$, to consider slack. The slack $S_{j}{ }^{+}=\max \left(d_{j}-p_{j}{ }^{*}-t, 0\right)$ for each job at time $t$ reflects its urgency relative to its due date $d_{j}$. If job $j$ has negative slack, meaning it will be tardy even if selected next, then $S_{j}^{+}=0$ and R\&M reduces to WSPT. However, if $S_{j}^{+}>0$, then its job priority is exponentially reduced as a function of slack. $p_{\text {ave }}$ is the average processing time of all jobs, and $k$ is a tuning parameter empirically derived based upon due date tightness.

We model the scheduler's aversion to risky jobs by applying a safety time ( $S T$ ) factor for prioritization purposes which, in turn, inflates the job processing times used for prioritization (Eq. 1) beyond their nominal times (of course, actual processing times are used to drive the simulation). Thus, the final processing time $p_{j}{ }^{*}$ used in Eq. 1 for prioritization is as follows:

$p_{j}^{*}=p_{j}\left[1+(1+S T) r_{j}\right]$

$r_{j}$ is the mean risk level. Parameter settings for these variables are provided in the next section.

The objective of this paper is to study and identify robust safety time values for use across a variety of cases involving the experimental factors and risk materialization scenario. 


\section{Experimental Design}

Discrete-event simulation is used to generate the experimental data. The design consists of $4 \times 2 \times 3 \times$ $3 \times 21=1512$ cases with each case replicated 250 times to provide robust estimates. Specifically, there are four major experimental cases investigated:

- Case 1: Risk-adjusted times (i.e., safety time) used in job prioritization, and the risk actually materializes.

- Case 2: Risk-adjusted times used in job prioritization, but risk does not materialize.

- Case 3: Risk-adjusted times not used in job prioritization, and risk does not materialize.

- Case 4: Risk-adjusted times not used in job prioritization, but risk does materialize.

Two mean risk levels $r_{j}$ are examined: 0.5 for low risk and 2.0 for high risk (non-risky jobs have a risk level of zero).

Three levels (percentage) of risky job levels are examined: $10 \%, 30 \%$ and $50 \%$ of the job stream contain "risky" jobs subject to processing time risk. The scheduler is assumed to know which jobs are risky and which jobs are not risky. A 25-job stream is used in all cases.

Three tardiness factor (TF) levels are studied: $0.1,0.4$ and 0.7 . TF represents the expected proportion of tardy jobs (e.g. $40 \%$ of jobs expected tardy when $T F=0.4$ ). After nominal processing times $p_{j}$ are generated for each job from a normal distribution with mean 20 and standard deviation 5 , the mean of the due date distribution $D D_{M E A N}$ is computed as $(1-T F)^{*} \sum p_{j}{ }^{*}$ where $\sum p_{j}{ }^{*}$ is the sum of the processing times (i.e., expected makespan). Individual job due dates $d_{j}$ are then generated from a normal distribution centered at $D D_{M E A N}$.

21 safety time factors (ST) ranging from -1.0 to +3.0 (in 0.2 increments) are studied. As stated, $S T$ is a factor used to model risk aversion behavior. High $S T$ values are used when high aversion exists. $S T=$ -1.0 corresponds to prioritizing jobs using only their nominal processing times (i.e., no risk aversion). $S T=0$ corresponds to prioritizing jobs using the mean risk level (e.g., at $r_{j}=0.5$ or 2.0 mean risk levels). $S T>0$ corresponds to utilizing additional risk time in job prioritization, such as when a scheduler is highly averse to particular risky jobs. Note ST only affects priorities for risky jobs; nonrisky jobs are prioritized using only their nominal times.

The fast machine is considered to be twice the speed of the slow machine. The performance objective used is weighted tardiness. Job weights are generated from a normal distribution with mean 40 and standard deviation 10 .

\section{Results and Discussion}

Appendix A contains the experimental results. Inner values represent total weighted tardiness across 250 replications for each factor combination. We begin by searching for robust safety time values (ST) in aggregate. Fig. 1 displays mean weighted tardiness results for Case 1 across the various safety time values. Recall Case 1 corresponds to when $S T$ is used for job prioritization and the job risk actually materializes. Results are aggregated across all risk levels, percent risky job levels and TF levels. The $S T$ interval yielding the best performance in aggregate is $[0,0.8]$ with the best value occurring at 0.6. 


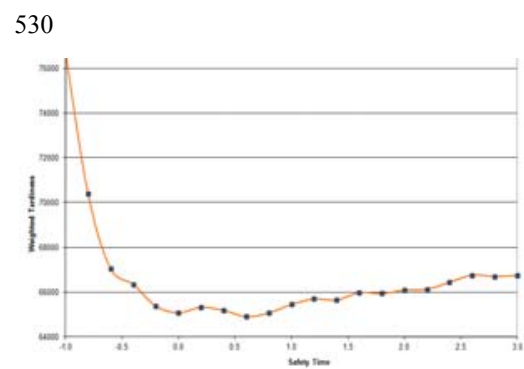

Fig. 1. Performance vs. Safety Time: Aggregate Across Factors (Risk Occurs)

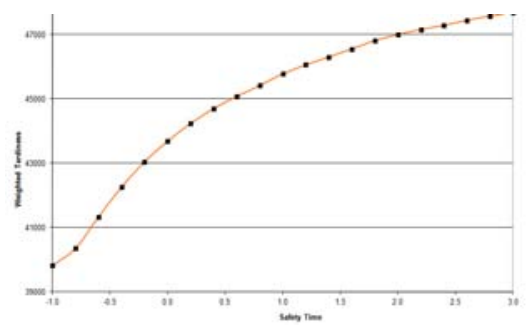

Fig. 2. Performance vs. Safety Time: Aggregate Across Factors (Risk Doesn’t Occur)

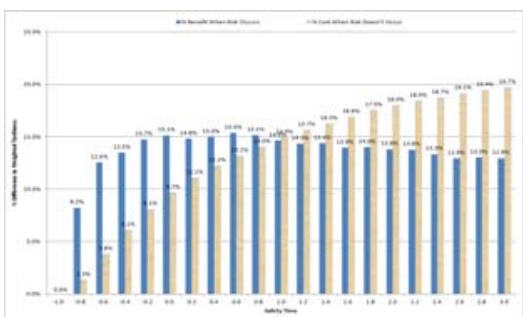

Fig. 3. Performance vs. Safety Time: Aggregate Across Factors

Fig. 2 displays total weighted tardiness results for Case 2. Case 2 corresponds to when ST is used for job prioritization, but the risk does not materialize. These results are shown primarily for confirmatory purposes since it is intuitive that, if the risk does not occur, then including no safety time should yield the best performance. Indeed, this is true since the best $S T$ value occurs at - 1.0, which corresponds to prioritizing jobs using only their nominal processing times.

Fig. 3 displays the percent performance increase (Cases 1 vs. 4) and decrease (Cases 2 vs. 3) when risk does and does not occur, respectively. These results are also aggregated across risk, percent risky jobs and tardiness factor levels. Performance gains exceeds losses within the ST interval, [- 0.8, 0.8], with $S T=0.6$ yielding the highest benefit. As expected, the percentage decrease in performance (i.e., "cost") associated with using safety time when the risk does not materialize rises as ST increases. These results are consistent with Fig. 1 and Fig. 2.

Let us now examine benefit-cost tradeoffs at various $S T$ values, which are important when uncertainty exists in whether or not the risk will occur. In such cases, the scheduler may ask, "What can I gain in performance vs. what can I lose?" Obviously, the "best" ST value will depend on the likelihood the risk will materialize. Referencing Fig. 3 at $S T=-0.8$, the benefit $(13.5 \%)$ is 6.3 times greater than the cost $(6.1 \%)$. For $S T=-0.4$, the benefit $(8.2 \%)$ is 2.2 times greater than $\operatorname{cost}(1.3 \%)$. At $S T=0$, benefit still exceeds cost by a factor of 1.55 . Recall $S T=0$ corresponds to prioritizing jobs using the expected risk times. For example, referencing Eq. 2, if nominal time is 20 minutes and risk level is 0.5 , the expected risk time is $20 \times 0.5=10$ minutes (one-half of nominal). If risk level is 2.0 , the expected risk time is $20 \times 2=40$ minutes ( 2 times nominal). If risk level 2.0 and $S T=1.0$, the planned risk time is 20 $\times 2 \times 2=80$ minutes (4 times nominal). Consequently, although $S T=0.6$ provides the greatest percent gain when the risk occurs, $S T=0.6$ or -0.8 may be "safer" values to use when uncertainty exists in whether the risk will materialize. These summary results also indicate that, when uncertainty exists, the mean performance gain when the risk occurs will significantly exceed the mean performance loss when it doesn't occur when using ST within the interval $[-0.8,0](\mathrm{p}=0.000035)$.

Thus far, we have only analyzed performance in aggregate. We now examine it at specific factor settings. Fig. 4 shows percent benefit-cost at the low risk level $\left(r_{j}=0.5\right)$. Here, performance gains exceed losses within a narrower $S T$ interval $[-0.8,0]$ than in the aggregate case. Moreover, gains and losses are lower than the aggregate case. It is interesting to note that performance gain when the risk occurs can become negative at very high $S T$ values $(S T \geq 2.4)$ implying that, under low risk, using too much safety time can result in worse performance than using none at all.

Fig. 5 shows benefit-cost relationships at the high risk level $\left(r_{j}=2.0\right)$. Performance gains and losses are higher than at the low risk scenario, which is intuitive. Also, gains exceed losses within a wider ST interval, $[-0.8,1.0]$ than the low risk and aggregate scenarios. 


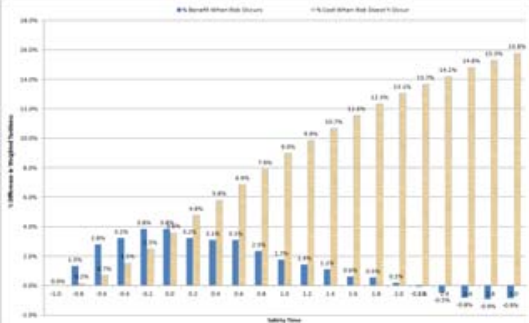

Fig. 4. Performance vs. Safety Time: Low Risk Level

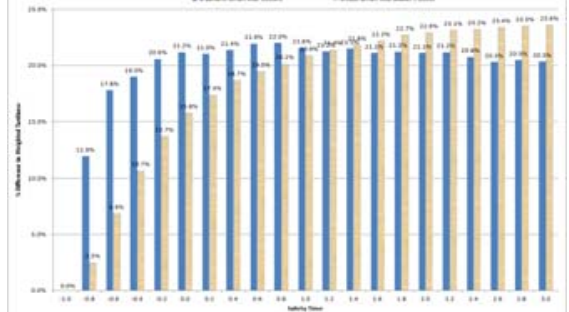

Fig. 5. Performance vs. Safety Time: High Risk Level

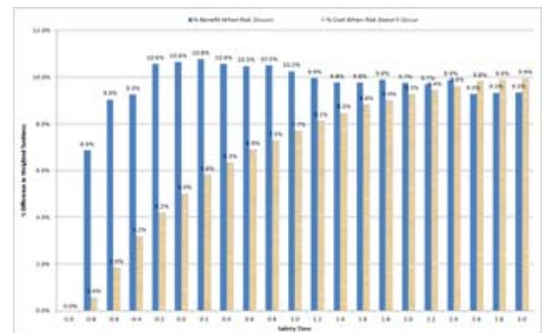

Fig. 6. Performance vs. Safety Time: 10\% Risky Jobs Level

Figs. 6-8 display benefit-cost relationships at the three percent risky job levels, $10 \%, 30 \%$ and $50 \%$, respectively. Performance gains and losses are lowest when only $10 \%$ of the jobs are risky (on average) and highest when $50 \%$ of the jobs are risky, which again is intuitive. However, it is interesting to note the ST interval within which gains exceed losses is much wider for $10 \%$ risky jobs than for $30 \%$ or $50 \%$. At $10 \%$ risky jobs, gains exceed losses for any $S T \leq 2.4$. Again, the "best" ST value to use will depend upon the likelihood the risk will materialize.

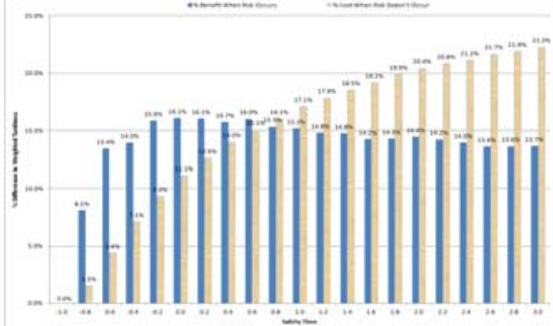

Fig. 7. Performance vs. Safety Time: $30 \%$ Risky Jobs Level

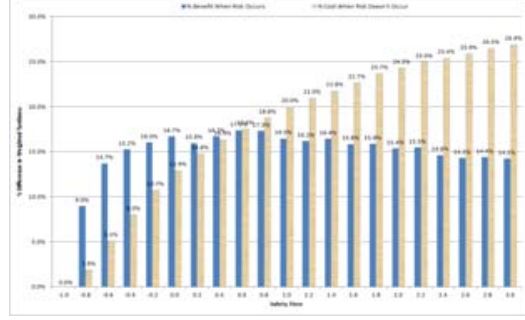

Fig. 8. Performance vs. Safety Time: $50 \%$ Risky Jobs Level

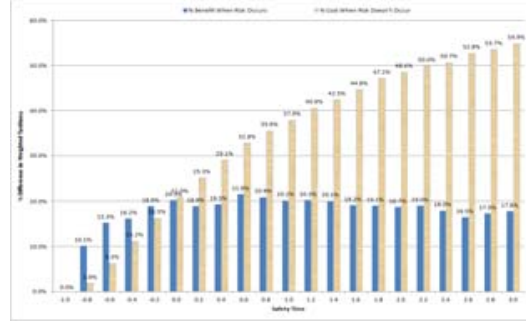

Fig. 9. Performance vs. Safety Time: 0.1 Tardiness Factor Level

Figs. 9-11 display benefit-cost relationships at the three tardiness factor levels, $0.1,0.4$ and 0.7 , respectively. In these cases, performance gains and losses are highest under the lowest TF setting (0.1), which is intuitive since more slack exists in the schedule relative to due dates for the R\&M rule to resequence jobs. However, the $S T$ interval within which gains exceed losses is much wider at the 0.7 level ( $70 \%$ tardy jobs on average) than at either the 0.1 or 0.4 levels.

Although weighted tardiness performance has been the focus thus far, we conclude this section by examining the rate at which risky jobs are offloaded to the slow machine. Fig. 12 displays the percent risky jobs processed on the slow machine in the scenarios when safety time is/is not utilized in priority assignments (Cases 1 and 4). In aggregate across all factors, $34.2 \%$ of risky jobs are offloaded to the slow machine when safety time was not used (and the equivalent case when ST $=-1$ ). This result is intuitive given the 2:1 speed advantage of the fast machine (i.e., $1 / 3$ of jobs on slow machine and 2/3 jobs on fast machine to balance makespan). When safety time is used, the percent risky jobs assigned to the slow machine increases to $40-45 \%$, depending on safety time.

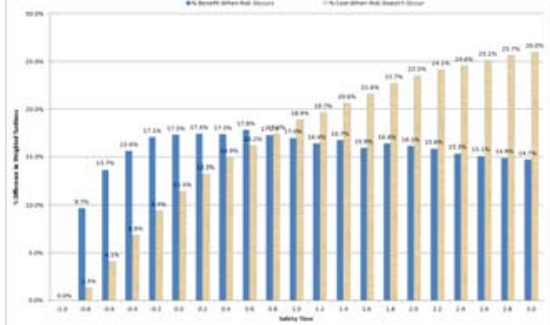

Fig. 10. Performance vs. Safety Time: 0.4 Tardiness Factor Level

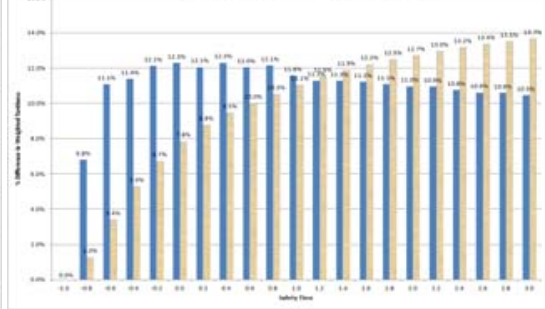

Fig. 11. Performance vs. Safety Time: 0.7 Tardiness Factor Level

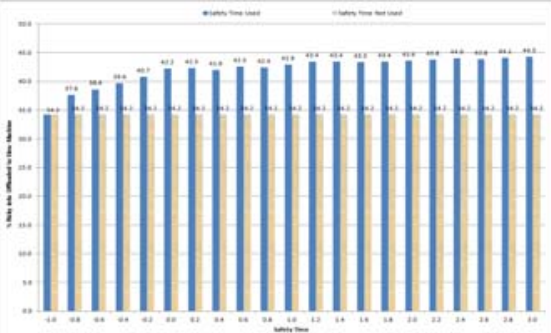

Fig. 12. Percent Risky Jobs on Slow Machine vs. Safety Time: Aggregate Across Cases 
Although graphs at specific risk, percent risk job and tardiness factor settings are not shown, the 40$45 \%$ rate is fairly consistent across those levels. This rate is reasonable given the proposition used to load the machines (Morton and Pentico, 1993) whereby the highest priority job is assigned to the machine expected to finish it first. Risky jobs are only shifted to the slow machine during times when the schedule is tight (i.e., peak load) and job slack is low.

\section{Conclusion and Future Directions}

This paper has extended the aversion dynamics research agenda from the single-machine environment to the parallel, proportional two- machine environment to study the effects of empirically observed risk mitigation strategies associated with offloading perceived risky jobs onto a secondary machine. A theoretical proposition (Morton and Pentico, 1993) was used to load the highest priority job on the machine that was expected to finish it first, and the R\&M bottleneck dynamics-based heuristic (Morton et al. 1995; Morton and Pentico, 1993) was used to compute job priorities. Four major cases were considered relative to the risk materializing or not and relative to whether or not safety time was applied to processing times within the R\&M rule to model risk aversion. Within each major case, two risk levels, three percent risky job levels and three tardiness factor levels were examined across a wide range of potential safety time values. Results indicated that performance gains achieved when risk materializes exceeds performance losses when risk does not materialize within specific safety time intervals. Performance gains and losses were highest at the high risk, high percent risky jobs and/or low tardiness factor settings. Moreover, the $S T$ interval within which gains exceed losses is widest at the high risk, low percent risky jobs and/or high tardiness factor settings. Lastly, we examined the percentage of risky jobs that were offloaded to the slow machine. Results indicated a substantially higher percentage of risky jobs were assigned to the slow machine when safety time was used, thus validating the intended risk aversion behavior under consideration.

Future directions in aversion dynamics research can take on various forms (McKay and Black, 2006; McKay, et al. 2002). The subject of this paper, the parallel machine environment, can be extended to consider more than the two machines with multiple speed factors. Further, these concepts can be extended to the general job shop or flow shop environments. Moreover, machine risk can be considered as opposed to job risk, thus placing the focus on specific machines as opposed to specific jobs. Furthermore, extensions to the anticipatory batch insertion research agenda can be pursued as detailed in Black, et al. (2008).

\section{Acknowledgements}

This paper would not have been possible without the long term encouragement and inspiration of Thomas Morton (Professor Emeritus - Carnegie Mellon University). He helped develop the initial foundations for Aversion Dynamics and provided the guidance and insights necessary to bring the ideas forward. Dr. Morton also provided valuable guidance as the parallel machine problem was formulated and the research planned.

\section{References}

Aytug, H, Lawley, M.A., McKay, K.N., Mohan, S., \& Uzsoy, R. (2005). Executing production schedules in the face of uncertainties: a review and some future directions. European Journal of Operations Research, 165, 86-110.

Black, G.W. (2001), Predictive, stochastic and dynamic extensions to aversion dynamics scheduling, Ph.D. dissertation, University of Alabama in Huntsville.

Black, G.W., McKay, K.N., \& Messimer, S.L. (2004). Predictive, stochastic and dynamic extensions to aversion dynamics scheduling. Journal of Scheduling, 7, 277-292.

Black, G.W., McKay, K.N., \& Messimer, S.L. (2005). Anti-fragmentation in aversion dynamics scheduling. International Journal on Production Research, 43, 109-129. 
Black, G.W., McKay, K.N., \& Morton, T.E. (2006). Aversion scheduling in the presence of risky jobs. European Journal of Operational Research, 175(1), 2006, 338-361.

Black, G.W., McKay, K.N., \& Varghese, S.E. (2008). 'Anticipatory batch insertion' to mitigate perceived processing risk. International Journal of Production Research, 46(4), 853-871.

Cao, Q., Patterson, J.W., \& Griffin, T.E. (2001). On the operational definition of processing time uncertainty. International Journal of Production Research, 39(13), 2833-2849.

Cowling, P., \& Johansson, M. (2002). Using real time information for effective dynamic scheduling. European Journal of Operational Research, 139, 230-244.

Dubois, D., Fargier, H., \& Fortemps, P. (2003). Fuzzy scheduling: modeling flexible constraints vs. coping with incomplete knowledge. European Journal of Operational Research, 147, 231-252.

Kanet, J.J., \& Sridharan, V. (2000). Scheduling with inserted idle time: problem taxonomy and literature review. Operations Research, 48(1), 99-110.

Kleijnen, J.P., \& Gaury, E. (2003). Short-term robustness of production management systems: a case study. European Journal of Operational Research, 148(2), 452-465.

McKay, K.N. (1992). Production planning and scheduling: a model for manufacturing decisions requiring judgment. Ph.D. Thesis, University of Waterloo.

McKay, K.N., Safayeni, F.R., \& Buzacott, J.A., (1995). Common sense realities of planning and scheduling in printed circuit board production. International Journal of Production Research, 33(6), 1587-1603.

McKay, K.N., \& Wiers, V.C.S. (1999). Unifying the theory and practice of production scheduling. Journal of Manufacturing Systems, 18(4), 241-255.

McKay, K.N., Morton, T.E., Ramnath, P., Wang, J. (2000). Aversion dynamics - scheduling when the system changes. Journal of Scheduling, 3, 71-88.

McKay, K.N., Pinedo, M., \& Webster, S. (2002). A practice-focused agenda for production scheduling research. Production and Operations Management, 11, 249-258.

McKay, K.N., \& Black, G.W. (2006). Aversion dynamics - adaptive production control heuristics incorporating risk. Journal of the Operations Research Society of Japan, 49(3), 152-173.

Morton, T.E., \& Pentico, D.W. (1993). Heuristic Scheduling Systems, New York: John Wiley \& Sons.

Morton, T.E., Narayan, V., Ramnath, P. (1995). A tutorial on bottleneck dynamics: a heuristic scheduling methodology. Production and Operations Management, 4(2), 94-107.

O’Donovan, R, Uzsoy R., \& McKay, K.N. (1999). Predictable scheduling and rescheduling on a single machine in the presence of machine breakdowns and sensitive jobs. International Journal of Production Research, 37, 4217-4233.

Ovackik, I.M., \& Uzsoy, R. (1994). Rolling horizon algorithms for single machine dynamic scheduling problem with sequence dependent setup times. International Journal of Production Research, 32, 1243-1263.

Ovackik, I.M., \& Uzsoy, R. (1995). Rolling horizon procedures for dynamic parallel machine scheduling with sequence dependent setup times. International Journal of Production Research, 33, 3173-3192.

Shafaei, R., \& Brunn, P. (1999). Workshop scheduling using practical (inaccurate) data Part 1: the performance of heuristic scheduling rules in a dynamic job shop environment using a rolling time horizon approach. International Journal of Production Research, 37(17), 3913-3925.

Shafaei, R., \& Brunn, P. (1999). Workshop scheduling using practical (inaccurate) data Part 2: an investigation of the robustness of scheduling rules in a dynamic and stochastic environment. International Journal of Production Research, 37(18), 4105-4117.

Shafaei, R., \& Brunn, P. (2000). Workshop scheduling using practical (inaccurate) data Part 3: a framework to integrate job releasing, routing and scheduling functions to create a robust predictive schedule. International Journal of Production Research, 38(1), 85-99. 
Appendix A: Summary of Experimental Results

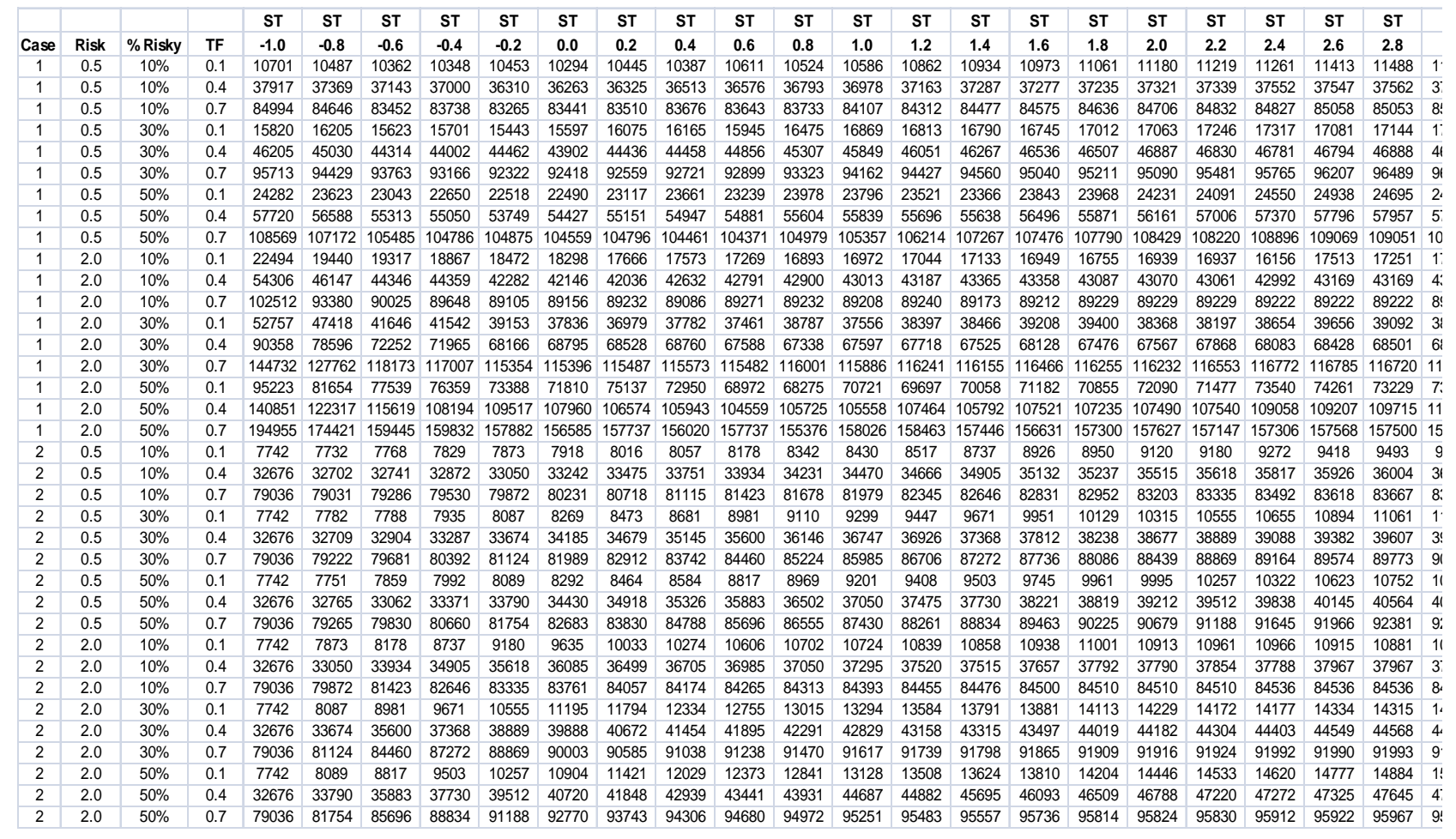

\begin{tabular}{|c|c|c|c|c|c|c|c|c|c|c|c|c|c|c|c|c|c|c|c|c|c|c|c|c|}
\hline & & & & ST & ST & ST & ST & ST & ST & ST & ST & ST & ST & ST & ST & ST & ST & ST & ST & & ST & ST & ST & \\
\hline Case & Risk & Risky & TF & -1.0 & -0.8 & -0.6 & -0.4 & & & 0.2 & 0.4 & 0.6 & 0.8 & 1.0 & 1.2 & & 1.6 & 1.8 & 2.0 & 2.2 & 2.4 & 2.6 & 2.8 & \\
\hline 3 & & & 0.1 & 7742 & 7742 & 7742 & 7742 & 7742 & 7742 & 7742 & 7742 & 7742 & 7742 & 7742 & 7742 & 42 & 7742 & 7742 & 7742 & 7742 & 7742 & 7742 & 7742 & \\
\hline & & & & & 32676 & & 32676 & 32676 & 32676 & 32676 & 32676 & 32676 & 32676 & 32676 & 32676 & 32676 & 32676 & 2676 & 32676 & 32676 & 32676 & 32676 & 2676 & \\
\hline & & & & & & & & & & & & & & & & & & & & & & & & \\
\hline 3 & & & & & 42 & & & 742 & & 7742 & 7742 & & & 7742 & 7742 & 7742 & 7742 & 7742 & 7742 & 7742 & & & & \\
\hline 3 & & & & & & & & & & & & & & & 32676 & & & & 676 & 2676 & & & & \\
\hline 3 & & & & & & & 336 & & & & & 036 & 036 & & & & & & & & & & & \\
\hline & & & & & & & & & & & & & & & & & & & 42 & & & & & \\
\hline 3 & & & & & 676 & & & & & & & & & & & & & & & & & & & \\
\hline & & & & & 36 & & b3 & & & & & & & & & & & & & & & & & \\
\hline & & & & & & & & & & & & & & & & & & & & & & & & \\
\hline & & & & & & & & & & & & & & & & & & & & & & & & \\
\hline & & & & & 3 & & 03 & & & & & & 6 & & & & & & & & & & & \\
\hline & & & & & & & & & & & & & & & & & & & & & & & & \\
\hline & & & & & & & & & & & & & & & & & & & & & & & & \\
\hline & & & & & & & & & & & & & 6 & & & & & & & & & & & \\
\hline & & & & & & & & & & & & & & & & & & & & & & & & \\
\hline & & & & & 7 & & & & & & & & & & & & & & & & & & & \\
\hline & & & & & & & & & & & & & & & & & & & & & & & & \\
\hline & & & & & & & & & & & & & & & & & & & & & & & & \\
\hline & & & & & & & & & & & & & & & & & & & & & & & & \\
\hline & & & & & & & & & & & & & & & & & & & & & & & & \\
\hline & & & & & & & & & & & & & & & & & & & & & & & & \\
\hline & & & & & & & & & & & & & & & & & & & & & & & & \\
\hline & & & & & & & & & & & & & & & & & & & & & & & & \\
\hline & & & & & & & & & & & & & & & & & & & & & & & & \\
\hline & & & & & & & & & & & & & & & & & & & & & & & & \\
\hline & & & & & 5569 & 10 & 10 & & & & & & 8569 & & & & & & & & & & & \\
\hline & & & & & & & & & & & & & & & & & & & & & & & & \\
\hline & & & & & & & & & & & & & & & & & & & & & & & & \\
\hline & & & & & & & & & & & & & & & & & & & & & & & & \\
\hline & & & & & & & & & & & & & & & & & & & & & & & & \\
\hline & & & & & & & & & & & & & & & & & & & & & & & & \\
\hline & & & & & & & & & & & & & & & & & & & & & & & & \\
\hline 4 & 2. & & & & 223 & & & & & & & & 9522 & & & & & & & & 9522 & & & \\
\hline & & & & & & & & & & & & & & & & & & & & & & & & \\
\hline 4 & 2.0 & $50 \%$ & 0.7 & 194955 & 4955 & 194955 & 194955 & 4955 & 4955 & 194955 & 34955 & 194955 & 194955 & 194955 & 194955 & 194955 & 19490 & 19450 & 19495 & 194955 & 194955 & 19495 & 19495 & \\
\hline
\end{tabular}

